\title{
Effetti del self-talk istruttivo e motivazionale sulle prestazioni cognitive e tecniche di giovani portieri di calcio
}

\author{
Effects of the instructional and motivational self-talk \\ on the cognitive and technical performance of \\ young soccer goalkeepers
}

\author{
Marco Guicciardi, Fabio Prinzis \\ Dipartimento di Pedagogia, Psicologia, Filosofia, \\ Facoltà di Studi Umanistici, Università degli Studi di Cagliari \\ via Is Mirrionis, 109123 Cagliari \\ e-mail: guicciardi@unica.it; telefono: 070.6757518 \\ e-mail: fabioprinzisfp195@gmail.com
}

Ricevuto: 28.04.2019 - Accettato: 29.09.2020

Pubblicato online: 31.05 .2021

\section{Riassunto}

Il dialogo interno (self-talk) è una modalità spesso utilizzata dagli atleti per far fronte alla competizione. La capacità di sapersi focalizzare su cue rilevanti per l'ottimale esecuzione del gesto tecnico può essere incrementata tramite l'utilizzo di affermazioni di auto-incoraggiamento o parole chiave. Tuttavia, se non opportunamente sostenuto, il dialogo interno può spesso sfociare in esclamazioni di auto-accusa o svalutazione che interferiscono negativamente con la prestazione. Questo studio sperimentale vuole valutare se le funzioni istruttive e motivazionali di un self-talk intenzionale possano influire sulle prestazioni cognitive e tecniche di giovani portieri di calcio. Dodici portieri militanti nelle categorie giovanissimi e allievi sono stati invitati ad eseguire sul campo di gioco, in modo controbilanciato inverso, due gesti tecnici: la parata e il rinvio. Successivamente

M. Guicciardi \& F. Prinzis./ Ricerche di Psicologia, 2020, Vol. 44, ISSN 0391-6081, ISSNe 1972-5620, doi:10.3280/rip2021oa11936 
è stato chiesto loro di utilizzare un self talk guidato di tipo motivazionale o istruttivo. Sono stati rilevati i livelli di efficacia e precisione prima e dopo l'utilizzo del self talk, unitamente alla frequenza e tipologia di pensieri interferenti. I risultati hanno mostrato un significativo miglioramento del rinvio, ma non della parata. Non sono emersi cambiamenti significativi nella frequenza dei pensieri interferenti. Il self-talk intenzionale può contribuire a consolidare l'esecuzione di alcuni specifici gesti tecnici e potrebbe diventare una strategia di intervento utile per la preparazione mentale dei giovani portieri.

Parole chiave: self-talk, pensieri interferenti, prestazioni, portieri, calcio

\begin{abstract}
Internal dialogue or self-talk is a mode often used by athletes to cope with the competition. The ability to focus on cue relevant for optimal execution of a technical movement can be increased by using self-talk or keywords. However, if not properly supported, internal dialogue can often result in statements of selfaccusation or devaluation which can negatively interfere with performance. This experimental study aims to assess whether the instructive and motivational functions of intentional self-talk can affect the cognitive and technical performance of young football goalkeepers. Twelve goalkeepers enrolled in the "under14/15" and "under16/17" categories were invited to perform two technical gestures on the soccer field in a counterbalanced manner: the goalkeeper save and the goal kick. Subsequently, they were asked to use either a motivational or instructive guided self-talk. The levels of effectiveness and precision were both measured before and after the use of self-talk, as well as the frequency and type of interfering thoughts. The results showed a significant improvement in the goal kick, but not in the goalkeeper save. No significant changes were found in the frequency of interfering thoughts. In conclusion, intentional self-talk may help to consolidate the execution of specific technical gestures and might become a useful intervention strategy for mental preparation of young goalkeepers.
\end{abstract}

Keywords: self-talk, interfering thoughts, performance, goalkeepers, football.

\title{
Introduzione
}

Il self-talk può essere definito come "un dialogo interno in cui gli individui interpretano $i$ sentimenti e le percezioni, regolano e cambiano le valutazioni e le conoscenze e si danno consigli e sostegno" (Hackfort \& Schvenkmezger, 1993). Spesso si è dibattuto se il dialogo interno soddisfi gli stessi requisiti sintattici e grammaticali delle produzioni discorsi- 
ve, pur essendo proferibile internamente o in forma sub-vocalica (Larrain \& Haye, 2012; Van Raalte, Vincent e Brewer, 2016). Allo stesso modo, ci si è interrogati in merito alla possibilità che il self-talk possa essere maggiormente profferto in modo spontaneo e automatico o in modo in14tenzionale e diretto ad uno scopo (Latinjak, Zourbanos, Lopez-Ros, 2014). Hardy, Oliver e Tod (2009) hanno sottolineato l'esigenza di un modello esplicativo che possa prendere in considerazione le complesse e reciproche relazioni che nello sport intercorrono tra self-talk, fattori personali (es. preferenze, convinzioni, etc.), fattori situazionali (es. difficoltà del compito, comportamenti dell'allenatore, etc.), meccanismi cognitivi (es. attenzione), motivazionali (es. fiducia in sé stessi), comportamentali (es. tecnica) e affettivi (es. ansia).

Tra i fattori che sono stati maggiormente studiati in ambito sportivo figurano: a) la valenza (positiva/negativa); b) la modalità espressiva (interna, sub-vocalica, esterna); c) la forma grammaticale (io, tu, noi); d) la funzione (istruttiva o motivazionale) (Hardy, 2006).

Per quanto riguarda la valenza positiva o negativa si è potuto osservare che gli atleti tendono ad utilizzare il self-talk per lodarsi o biasimarsi, motivarsi a raggiungere i propri obiettivi, gestire i pensieri e le emozioni interferenti e per regolare le interazioni sociali. La valenza positiva o negativa del dialogo interno varia in funzione degli eventi: quando accadono eventi positivi (es. vittoria) gli atleti sono propensi a lodarsi, mentre di fronte ad una sconfitta e più probabile che il biasimo prenda il sopravvento. Sono stati documentati anche gli effetti del self-talk positivo o negativo sulla successiva prestazione: coloro cui si chiedeva di auto-incitarsi con parole positive (es. "posso farlo"), riportavano migliori prestazioni nel lancio delle freccette rispetto a coloro che dovevano utilizzare parole di auto-svalutazione ("non posso farlo") o al gruppo di controllo (Van Raalte Brewer, Lewis, Linder, et al.,1995). Tuttavia, non sempre vi è una corrispondenza biunivoca tra risultato e valenza del self-talk o viceversa. Difatti frasi con valenza positiva possono avere degli effetti debilitanti quando distraggono dal compito e viceversa frasi con valenza negativa possono svolgere una funzione facilitante, stimolando l'atleta a migliorare il suo rendimento (Hardy, Gammage, \& Hall, 2001; Theodorakis, Hatzigeorgiadis \& Zourbanos, 2012).

Per quanto riguarda la modalità espressiva Hardy (2006) ha evidenziato che il self-talk può essere prodotto in maniera udibile, a bassa voce, in forma sub-vocalica o completamente interna. Ulteriori studi dovrebbero testare in ambito sportivo l'efficacia comparativa di ciascuna modalità espressiva (Van Raalte, et al., 2016). 
Relativamente alla forma grammaticale è stato osservato che spesso le persone, quando stanno eseguendo un compito, possono riferirsi a sé stesse usando il pronome in prima persona singolare "io", in seconda persona "tu" o in prima persona plurale "noi". Coloro che utilizzano preferibilmente il "tu" o il "noi" sembrano ottenere migliori risultati in condizioni che richiedono un'autoregolazione consapevole, rispetto a coloro che utilizzano il pronome "io" (Dolcos e Albarracin, 2015). Sembrerebbe infatti che l'utilizzo del self-talk in prima persona porti a valutare come minacciose le situazioni stressanti (Kross, Bruehlman-Senecal, Park, Burson, Dougherty, Shablack, 2014). Tali considerazioni necessitano tuttavia di maggiori approfondimenti in ambito sportivo (Van Raalte et al., 2016).

A partire dal lavoro seminale di Hardy (2006), la funzione istruttiva o motivazionale è stata ampiamente studiata in differenti discipline sportive come il golf (Harvey, Van Raalte e Brewer, 2002), il tennis (Van Raalte, Brewer, Rivera, \& Petitpas, 1994), il basket (Perkos, Theodorakis e Chroni, 2002; Chroni, Perkos \& Theodorakis, 2007), hockey su ghiaccio (Rogerson \& Hrycaiko, 2002) e il calcio (Farina \& Cei, 2019). In una meta-analisi pubblicata da Hatzigeorgiadis, Zourbanos, Galanis e Theodorakis (2011) il self-talk istruttivo è risultato più efficace di quello motivazionale nel migliorare le prestazioni in compiti di precisione e nella esecuzione di compiti nuovi, rispetto a quelli più familiari. Theodorakis, Weinberg, Natsis, Douma e Kazakas (2000) hanno ipotizzato che per le attività che richiedono abilità, tempismo e precisione, il self-talk istruttivo sia più utile poiché facilita l'esecuzione dei movimenti desiderati e porta il focus attentivo sui segnali rilevanti per la corretta esecuzione del compito.

Affinché il self-talk svolga questa funzione è necessario che le parole siano: a) brevi e specifiche; b) foneticamente semplici; c) correlate all'attività in corso; d) compatibili con il timing dell'azione; e) formulate in prima persona e al presente; f) ripetute spesso e in anticipo; g) con valenza positiva; h) apprese e allenate come elementi di richiamo per fronteggiare i momenti più stressanti (Landin, 1994; Farina \& Cei, 2019).

Viceversa, il self-talk motivazionale ha dato migliori risultati nei compiti che richiedono una coordinazione grosso-motoria, come ad esempio nel getto del peso o nelle discipline di resistenza e lunga durata. Il self-talk motivazionale può favorire un miglior controllo dei livelli di attivazione, un maggior rilassamento o un incremento dello sforzo in funzione delle fasi della competizione (Hatzigeorgiadis, Theodorakis \& Zourbanos, 2004). Entrambe le tipologie di self-talk producono migliori 
risultati con la pratica e si sono dimostrate utili anche nel recupero da infortuni (Beneka, Malliou, Gioftsidou, Kofotolis, Rokka, Mavromoustakos et al., 2013).

Per quanto riguarda i metodi di studio, due sono state le strategie di ricerca maggiormente utilizzate: gli esperimenti di laboratorio e le indagini correlazionali.

Per quanto riguarda gli esperimenti di laboratorio si è cercato di riprodurre in condizioni rigorosamente controllate semplici gesti tecnici o compiti motori semplificati. In base alle tipologie intenzionali di selftalk, di tipo istruttivo o motivazionale, assegnate casualmente dallo sperimentatore, si sono osservati i risultati ottenuti sulla successiva prestazione degli atleti (Theodorakis et al., 2012).

Le indagini correlazionali si sono focalizzate sulla percezione dell'uso spontaneo del self-talk da parte degli atleti, in allenamento e in gara, facendo riferimento alla sua frequenza d'uso in differenti discipline sportive (Hardy, 2006). Si è potuto riscontrare che il self-talk viene utilizzato prevalentemente durante la gara, piuttosto che in allenamento, ma vi sono evidenze del suo utilizzo anche prima e dopo la competizione sportiva (Hardy et al., 2001). Gli atleti di sport individuali e gli atleti più esperti sembrerebbero utilizzare più frequentemente il self-talk rispetto agli atleti che praticano sport di squadra e agli atleti meno esperti. La frequenza d'uso del self-talk sembra aumentare in relazione al periodo della stagione agonistica e rispetto al momento della gara (Hardy \& Hall, 2004).

A nostra conoscenza un solo studio di tipo correlazionale ha indagato la percezione degli effetti positivi o negativi del self-talk sulla successiva prestazione in calciatori, nella fattispecie iraniani a livello di squadra nazionale (Daftari, Sofian, Omar-Fauzee, Akbari, 2010). I risultati ottenuti testimoniano una prevalenza di effetti positivi quali il miglioramento della coordinazione con i compagni, della focalizzazione dell'attenzione e del processo decisionale. Tra gli effetti negativi sono stati citati l'aumento dello stress e l'indebolimento della fiducia in sé stessi dovuto ad un eccesso di auto ed etero criticismo.

Con l'obiettivo di contribuire ad una maggiore conoscenza degli effetti che l'uso intenzionale del self-talk può produrre sulla prestazione tecnica e mentale dei giovani calciatori, abbiamo condotto uno studio sperimentale con intervento, per valutare quanto il self-talk, istruttivo o motivazionale, influenzi le prestazioni dei portieri in due compiti specifici: parata e rinvio. Infine, abbiamo inteso verificare se l'utilizzo consapevole del self-talk possa influenzare la frequenza di pensieri distraenti accorsi durante la sperimentazione. 


\section{Metodo}

\section{Panoramica}

È stato utilizzato un disegno di ricerca entro i soggetti, in cui in ordine controbilanciato inverso ciascun partecipante è stato esposto alle due condizioni di stimolazione (self-talk istruttivo vs. motivazionale), in relazione a due compiti tecnici (parata vs. rinvio), secondo un disegno a misure ripetute del tipo pre-post con intervento per un totale di 6 sessioni complessive.

\section{Partecipanti}

Hanno partecipato alla ricerca un gruppo di 12 portieri maschi di età compresa tra 14 e 17 anni $(\mathrm{M}=15$; $\mathrm{DS}=1.21)$, con una esperienza media nel ruolo di portiere di 4 anni e 8 mesi ed un volume di allenamento settimanale in media pari a 11.2 ore. Tutti i portieri di nazionalità italiana erano tesserati con la Federazione Italiana Gioco Calcio (FIGC), e militavano in campionati dilettantistici regionali. Nessuno dei portieri aveva riportato infortuni negli ultimi 4 mesi.

A tutti i partecipanti in quanto minorenni, è stato chiesto loro di far firmare un foglio di consenso informato ad entrambi i genitori ed è stata ribadita la possibilità di poter abbandonare lo studio sperimentale in qualsiasi momento senza dover giustificare tale scelta. Un solo portiere non ha portato a termine tutte le sessioni previste e pertanto i suoi dati sono stati eliminati dalle analisi successive.

\section{Procedura}

Lo studio sperimentale è stato condotto su un campo di gioco e si è svolto in un arco di tempo di otto settimane. Tutti i compiti sono stati effettuati con cadenza settimanale il lunedì pomeriggio sullo stesso terreno in erba dai giovani portieri, equipaggiati con scarpe da calcio (tacchetti in gomma) e guanti appropriati.

\section{Fase pre}

In questa fase i portieri riuniti in gruppi di quattro hanno effettuato quindici minuti di riscaldamento e successivamente hanno affrontato il compito uno alla volta. Dopo due tiri di prova, uno lanciato a destra del portiere e l'altro alla sua sinistra, da una macchina spara palloni (Globus Eurogoal 1500) posizionata a 20.5 metri di distanza dal centro della porta e con una potenza pari al del $67 \%$ della potenza massima, venivano lanciati una sequenza di dieci palloni. Di questi, i primi cinque venivano tirati alla destra del portiere, i restanti cinque alla sua sinistra. Per ciascun 
tiro sono stati assegnati i seguenti punteggi: 1 punto nel caso di parata, 0.5 punti se la palla toccava il corpo del portiere ma entrava in rete, 0 punti in caso di goal.

Il compito di rinvio consisteva nel colpire una sagoma posta a 33.5 metri di distanza calciando un pallone fermo a terra. A fianco alla sagoma son stati posti due paletti rispettivamente a distanza di un metro a destra e a sinistra. Dopo due rinvii di prova i ragazzi hanno effettuato dieci rinvii a palla ferma tutti dalla stessa posizione utilizzando il loro piede dominante. Per ciascun portiere son stati registrati i seguenti punteggi: 1 punto se la sagoma veniva colpita, 0.5 punti se la palla passava in mezzo ai paletti, 0 punti se la palla veniva calciata al di fuori dei paletti.

Dopo il compito a ciascun portiere son stati consegnati i due seguenti questionari: Il Thought Occurrence Questionnaire for Sport (TOQS) di (Hatzigeorgiadis \& Biddle, 2000) e il questionario SF1 da noi predisposto.

Il TOQS misura la frequenza dei pensieri intrusivi con tre scale: preoccupazioni relative alla gara, pensieri irrilevanti rispetto alla situazione e pensieri di fuga. Il test si compone di diciassette affermazioni che richiedono di riferire i pensieri avuti in gara nelle ultime quattro settimane (Ad esempio: ho pensato ai miei precedenti errori; che le condizioni meteo non sono buone; che non vinceremo questa partita, etc.). La scala di risposta valuta la frequenza, da 1 (quasi mai) a 7 (molto spesso), con cui tali pensieri si sono manifestati. Il test è stato validato con atleti adolescenti (Lane, Harwood \& Nevill, 2005). La versione italiana è stata predisposta seguendo una procedura di back translation.

Il questionario SF1 si compone di quattro quesiti tesi a valutare l'uso spontaneo del self-talk da parte dei giovani portieri. Le domande di tipo aperto erano le seguenti: 1) Durante una partita ti capita mai di parlare a te stesso? 2) In quali circostanze? 3) Che cosa ti sei detto? 4) Che effetto ha avuto questo dialogo?

\section{Intervento}

Nel secondo giorno di prove a tutti i giovani portieri è stato spiegato brevemente l'uso del self-talk e la sua funzione motivazionale e istruttiva.

Ai ragazzi son state fornite due frasi di self-talk motivazionali: "La paro!" (parata), e "La colpisco!" (rinvio) e due frasi di self-talk istruttivo: "Passo e spingo!" (parata) e "Mira alla sagoma mentre rinvii!" (rinvio).

Infine, è stato chiesto ai portieri nei quattro successivi incontri, prima di eseguire ciascun gesto tecnico, di ripetere ad alta voce o in modo subvocalico le due frasi. 
Tale procedura ha consentito di far sì che tutti i portieri svolgessero entrambi i compiti in tutte le permutazioni possibili. Ogni sessione ha avuto una durata media di $15^{\prime}$ per portiere.

\section{Fase post}

Nella fase post i giovani portieri ragazzi hanno effettuato i due compiti tecnici della parata e del rinvio senza ricevere alcuna istruzione in merito all'uso del self-talk. Al termine delle sequenze hanno compilato nuovamente il TOQS e un questionario SF2 volto a valutare sulla base della letteratura esistente: a) la frequenza di utilizzo del self-talk nel corso dell'esperimento b) gli effetti percepiti del suo utilizzo e c) la preferenza per una tipologia o l'altra (Chroni et al., 2007; Weinberg \& Gould, 2003; Zinsser, Bunker \& Williams, 2001). Relativamente al primo aspetto il questionario poneva due domande: "Con quale frequenza hai ripetuto le parole '...', durante il rinvio?" e "Con quale frequenza hai ripetuto le parole '...', durante la parata?". Ogni portiere poteva rispondere utilizzando una scala Likert a 7 gradini da " $1=$ mai" a "7 = sempre". Per quanto riguarda gli effetti percepiti, utilizzando un continuum che andava da "per niente" a "moltissimo" i portieri hanno risposto alle seguenti domande: "pensi che le parole '...' che hai usato ti abbiano aiutato a: a) concentrarti meglio; b) sentirti più sicuro; c) sentirti più forte; d) sentirti più rilassato; e) eseguire meglio la tecnica". Infine, è stato chiesto ai portieri "Quale dei due tipi di self-talk ti è piaciuto di più per la parata?" "Quale dei due tipi di self-talk ti è piaciuto di più per il rinvio?". Anche in quest'ultimo caso i partecipanti hanno risposto utilizzando una scala Likert a 7 punti per ciascuna delle tipologie di self-talk.

\section{Analisi dei dati}

L'analisi preliminare dei dati condotta con il test di KolmogorovSmirnov e correzione di Lilliefors ha confermato una distribuzione normale per tutti i parametri considerati. Pertanto, le successive analisi sono state condotte con $t$ test a misure ripetute per valutare gli eventuali cambiamenti nell'esecuzione dei due gesti tecnici e nella frequenza dei pensieri intrusivi. L'efficacia differenziale della tipologia di self-talk è stata valutata tramite una ANOVA fattoriale a misure ripetute del tipo $2 \mathrm{X} 4$, con successiva analisi dei trend 


\section{Risultati}

Per quanto riguarda gli effetti del self-talk sul miglioramento dell'esecuzione dei due gesti tecnici sono emerse differenze statisticamente significative tra pre e post-intervento in merito al rinvio $\left(t_{(10)}=-2,72, \mathrm{p}<\right.$. $05)$. Viceversa, nella parata non si sono osservati significativi miglioramenti $\left(t_{(10)}=-, 14\right.$, ns.) (Fig 1).

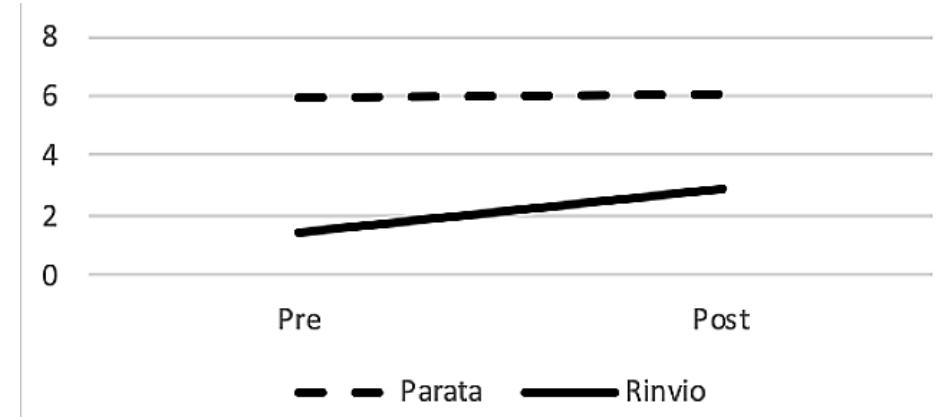

Fig. 1 - Media prestazioni nei due compiti tecnici nel pre e post-intervento

Considerando la prestazione dei singoli portieri nel rinvio, abbiamo osservato (Fig. 2) che un solo portiere ha peggiorato la sua prestazione. Viceversa, a parte tre portieri che hanno mantenuto la prestazione costante, tutti gli altri hanno migliorato il rinvio. Due portieri in particolare (n. 2 e n. 4) hanno mostrato notevoli miglioramenti.

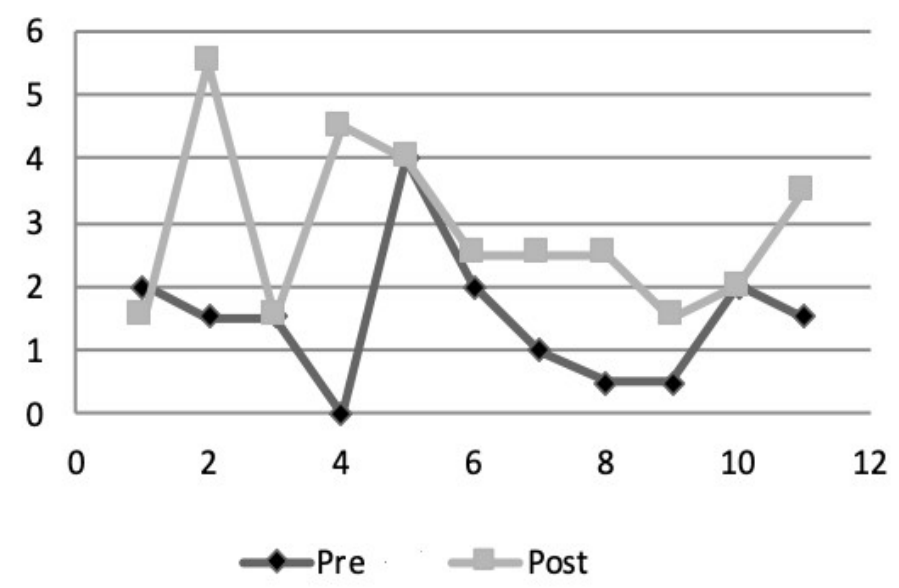

Fig. 2 - Prestazioni individuali nel rinvio nel pre e post-intervento 
Confrontando l'andamento delle prestazioni nel rinvio in relazione alle due tipologie di self-talk (Fig. 3) è emerso che solo il self-talk istruttivo ha prodotto un miglioramento lineare $\left(\mathrm{F}_{(1 ; 10)}=8,4 ; \mathrm{p}<.05\right)$; il selftalk motivazionale, pur essendo associato inizialmente a prestazioni migliori ha nel corso delle sessioni manifestato un andamento irregolare portando agli stessi risultati finali.

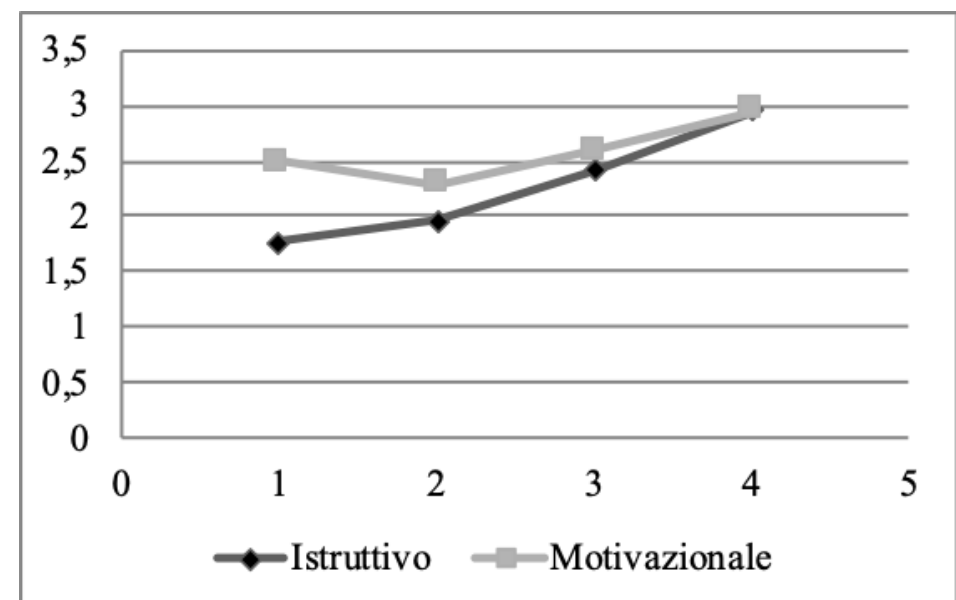

Fig. 3 - Andamenti delle prestazioni del rinvio in funzione della tipologia di self-talk

Per quanto riguarda i pensieri intrusivi misurati con il TOQS, seppure siano diminuiti a seguito dell'intervento, essi non sono risultati in media, significativamente inferiori rispetto a quelli manifestati prima dell'utilizzo del self-talk $\left(t_{(10)}=, 65\right.$, ns.).

Relativamente alla percezione dell'uso del self-talk, il questionario somministrato prima dell'intervento (SF1) ha evidenziato che i portieri avevano una bassa consapevolezza dell'utilizzo del self-talk durante le gare. Sette portieri hanno dichiarato di non utilizzare un dialogo interno durante le partite. Tre portieri utilizzavano il self-talk solo dopo un errore per rimproverarsi o caricarsi, mentre uno lo utilizzava per complimentarsi con sé stesso dopo una parata.

Dai dati raccolti alla fine dell'intervento si è potuto osservare tramite il questionario SF2 che i partecipanti hanno fatto un uso frequente del self-talk $(70 \%)$, con una leggera preferenza nel compito di parata $(74 \%)$ rispetto a quello di rinvio $(66 \%)$.

I portieri che hanno fatto un uso più frequente della tecnica del selftalk, hanno ammesso a livello personale che la tecnica è servita loro per aumentare la concentrazione, sentirsi più sicuri e rilassati e per eseguire meglio il gesto motorio. 
Infine, è emerso che in genere il self-talk motivazionale è stato apprezzato di più rispetto a quello istruttivo, in particolare nel compito di parata. Il self-talk istruttivo invece è stato viceversa apprezzato maggiormente per il compito di rinvio.

\section{Conclusioni}

Il self-talk da risorsa per l'atleta può diventare un elemento di disturbo quando distragga dal compito, risulti troppo frequente o interferisca con l'esecuzione automatica del gesto atletico. Pertanto, il ritmo e il timing del gesto atletico dovrebbero essere presi in considerazione al fine di ridurre la possibilità che le parole possano interferire con il flusso della prestazione

Nel calcio sono molto rari gli studi che hanno indagato empiricamente gli effetti del self-talk sulla prestazione (Farina \& Cei, 2019). In particolare, l'unico studio che a nostra conoscenza è stato condotto è di tipo correlazionale e si è focalizzato sulla percezione degli effetti del selftalk, di tipo facilitante o inibente, coinvolgendo calciatori adulti di livello nazionale.

Lo scopo principale del presente studio è stato quello di esplorare gli effetti di due tipologie di self-talk (istruttivo e motivazionale) sulle prestazioni della parata e del rinvio e sulla frequenza di pensieri interferenti in giovani portieri di calcio.

Per quanto riguarda il rinvio l'intervento attuato ha prodotto un significativo miglioramento nell'esecuzione del gesto tecnico. In particolare, il self-talk istruttivo si è dimostrato efficace nel migliorare sessione per sessione la precisione del tiro. Viceversa, il self-talk motivazionale pur avendo dato un iniziale riscontro superiore a quello istruttivo non ha manifestato un eguale incremento nelle sessioni successive.

Questo risultato conferma quanto riportato in letteratura a proposito dei migliori benefici del self-talk istruttivo, rispetto a quello motivazionale, nei compiti che richiedono un buon grado di precisione (Hatzigeorgiadis et al., 2011; Perkos et al., 2002).

In merito alla parata, l'uso di entrambe le tipologie di self-talk non ha prodotto risultati particolarmente soddisfacenti. La mancanza di risultati statisticamente significativi potrebbe essere attribuita alla presenza di un effetto tetto dal momento che tutti i giovani portieri hanno manifestato sin dalla fase iniziale una buona padronanza di questo gesto tecnico.

Contrariamente alle ipotesi, l'utilizzo deliberato del self-talk sembra non avere influito sulla frequenza di pensieri intrusivi, rilevati tramite il TOQS. Tale risultato necessita di ulteriori approfondimenti. È probabile che il differente contesto di riferimento (sperimentazione vs. competizio- 
ne) abbia reso meno salienti alcune caratteristiche della prestazione, senza influire sul livello di attivazione dei giovani portieri, che hanno sperimentato con minore intensità emotiva gli effetti delle loro prestazioni.

A livello qualitativo i giovani portieri che hanno fatto un maggior uso del self-talk hanno identificato nell'aumento della concentrazione, della fiducia in sé e nel miglioramento del gesto tecnico, i benefici maggiormente percepiti.

Lo studio presenta alcuni limiti. Innanzitutto, l'esigua numerosità del campione può avere portato a sottostimare l'ampiezza di alcuni effetti, risultati statisticamente non significativi. Inoltre, l'adesione volontaria e la scelta nel reclutamento da parte dei loro allenatori può avere introdotto un bias nella selezione dei partecipanti, che riduce la generalizzabilità dei risultati ottenuti. Infine, il diverso rendimento nel compito di rinvio rispetto a quello di parata, oltre ad un effetto tetto già discusso, potrebbe essere dovuto ad una maggiore familiarità del gesto tecnico così come all'incitamento formulato in seconda persona ("Mira alla sagoma mentre rinvii"), che, rispetto alle frasi formulate in prima persona, può avere ridotto il carico ansiogeno derivante dagli errori precedentemente commessi.

In conclusione, il self-talk può svolgere una funzione proattiva e migliorare nei giovani portieri l'efficacia di alcuni gesti tecnici ancora da consolidare (es. rinvio). In particolare, il self-talk di tipo istruttivo risulta più efficace nel migliorare un gesto che richiede precisione. Viceversa, rimane da chiarire il contributo del self-talk nel rafforzamento di gesti tecnici bene appresi e/o che richiedono una buona coordinazione motoria (es. parata). Infine, sarebbe opportuno approfondire con successive indagini la capacità del self-talk di interrompere nei giovani portieri, che stanno fronteggiando situazioni molto stressanti (es. partita), l'irruzione di pensieri interferenti.

\section{Riferimenti bibliografici}

Beneka, A., Malliou, P., Gioftsidou, A., Kofotolis, N., Rokka, S., Mavromoustakos, S., \& Godolias, G. (2013). Effects of instructional and motivational self-talk on balance performance in knee injured. European Journal of Physiotherapy, 15, 56-63. DOI: 10.3109/21679169.2013.776109.

Chroni, S., Perkos, S., \& Theodorakis, Y. (2007). Function and preferences of motivational and instructional self-talk for adolescent basketball players. Athletic Insight, 9(1), 19-31.

Daftari, O. Sofian, O. F. M., Omar-Fauzee, M. F., \& Akbari, A. (2010). Effects of Self-Talk on Football Players. Performance Sport Science Quarterly, 1(1), 29- 37. 
Dolcos, S., \& Albarracin, D. (2015). The inner speech of behavioral regulation: intentions and task performance strengthen when you talk to yourself as a you. European Journal of Social Psychology, 44, 636-642. DOI: 10.1002/ejsp.2048.

Farina, M. \& Cei, A. (2019). Concentration and self-talk in football. In In: E. Konter, J. Beckmann, J., \& T. Loughead, T. (Eds.), Football Psychology: From Theory to Practice. (pp.241-254). London, UK: Routledge. DOI: 10.4324/9781315268248-19.

Hackfort, D., \& Schwenkmezger, P. (1993). Anxiety. In R.N. Singer, M. Murphey, \& L.K. Tennant (Eds.), Handbook of Research on Sport Psychology (pp. 328-364). New York: Macmillan.

Hardy, J., Gammage, K., \& Hall, C. (2001). A descriptive study of athlete selftalk. The Sport Psychologist, 15, 306-318. DOI: 10.1123/tsp.15.3.306.

Hardy, J. \& Hall, C., (2004). A note on athletes' use of self-talk. Journal of Applied Sport Psychology, 16, 251-257. DOI: 10.1080/10413200490498357.

Hardy, J. (2006). Speaking clearly: a critical review of the self-talk literature. Psychology of Sport and Exercise, 7, 81-97. D O I: 10.1016/j.psychsport.2005.04.002.

Hardy, J., Oliver, E., \& Tod, D. (2009). A framework for the study and application of self-talk within sport. In: S. Mellalieu \& H. Sheldon (Eds.). Advances in Applied Sport Psychology a review (pp. 37-74). New York, NY: Routlege

Harvey, D.T., Van Raalte, J.L., \& Brewer, B.W. (2002). Relationship between self-talk and golf performance. International Sports Journal, 6, 84-91.

Hatzigeorgiadis, A., \& Biddle, S. J. H. (2000). Assessing cognitive interference in sport: development of the Thought Occurrence Questionnaire for Sport (TOQS). Anxiety, Stress \& Coping: An International Journal, 13, 65-86. DOI: $10.1080 / 10615800008248334$.

Hatzigeorgiadis, A., Theodorakis, Y., \& Zourbanos, N. (2004). Self-talk in the swimming pool: The effects of self-talk on thought content and performance on water polo tasks. Journal of Applied Sport Psychology, 16, 138-150. DOI: $10.1080 / 10413200490437886$.

Hatzigeorgiadis, A. Zourbanos, N. Galanis, E., \& Theodorakis, G. (2011). Selftalk and sports performance: A meta-analysis perspectives. Psychological Science, 6(4), 348-356. DOI: 10.1177/1745691611413136.

Kross, E., Bruehlman-Senecal, E., Park, J., Burson, A., Dougherty, A., Shablack, H., Bremner, R., Moser, J., \& Ayduk, O. (2014). Self-talk as a regulatory mechanism: how you do it matters. Journal of Personality and Social Psychology, 106, 304-324. DOI: 10.1037/a0035173.

Landin, D. K. (1994). The role of verbal cues in skill learning. Quest, 46, 299313. DOI: 10.1080/00336297.1994.10484128.

Lane, A. M., Harwood, C., \& Nevill, A. M. (2005). Confirmatory factor analysis of the Thought Occurrence Questionnaire for Sport (TOQS) among adolescent atlete. Anxiety, Stress, and Coping, 18(3), 245-2 54. DOI: 10.1080/10615800500134266.

Larrain, A., \& Haye, A. (2012). The discursive nature of inner speech. Theory \& Psychology, 21(1), 3-2. DOI: 10.11772F0959354311423864. 
Latinjak, A.T., Zourbanos, N., \& Lopez-Ros, V. (2014). Goal-directed and undirected self-talk: Exploring a new perspective for the study of athletes' selftalk, Psychology of Sport and Exercise 15, 548-558. DOI: 10.1016/j.psychsport.2014.05.007.

Perkos, S., Theodorakis, Y., \& Chroni, S. (2002). Enhancing performance and skill acquisition in novice basketball players with instructional self-talk. The Sport Psychologist, 16, 368-383. DOI: 10.1123/tsp.16.4.368.

Rogerson, L. J., \& Hrycaiko, D. W. (2002). Enhancing competitive performance of ice hockey goaltenders using centering and self-talk. Journal of Applied Sport Psychology, 14, 14-26. DOI: 10.1080/10413200209339008.

Theodorakis, Y., Weinberg, R., Natsis, P., Douma, E., \& Kazakas, P. (2000). The effects of motivational versus instructional self-talk on improving motor performance.The Sport Psychologist, 14, 253-272. DOI: 10.1123/tsp.14.3.253.

Theodorakis, Y., Hatzigeorgiadis, A., \& Zourbanos, N. (2012). Cognitions: selftalk and performance. In S. Murphy (Ed.), The Oxford handbook of sport and performance psychology (pp. 191-212). New York: Oxford University Press. DOI: 10.1093/oxfordhb/9780199731763.013.0010.

Van Raalte J. L., Brewer, B. W., Rivera, P. M., \& Petitpas, A. J. (1994). The relationship between observable self-talk and competitive junior tennis players' match performances. Journal of Sport and Exercise Psychology, 16, 400415. DOI: 10.1123 jsep.16.4.400.

Van Raalte, J. L., Brewer, B. W., Lewis, B. P., Linder, D. E., Wildman, G. \& Kozimor, J. (1995). Cork! The effects of positive and negative self-talk on dart throwing performance. Journal of Sport Behavior, 18(1), 50-57.

Van Raalte, J.L., Vincent, A., \& Brewer, B. (2016). Self-talk: review and sportspecific model. Psychology of Sport and Exercise, 22, 139-148. DOI: 10.1016/j.psychsport.2015.08.004.

Weinberg, R. S., \& Gould, D. (2003). Foundations of Sport and Exercise Psychology. Champaign, IL: Human Kinetics.

Zinsser, N., Bunker, L. K, \& Williams, J. M. (2001). Cognitive techniques for improving performance and building confidence. Applied Sport Psychology: Personal Growth to Peak Performance (4th ed., pp. 284-311). Mountain View, CA: Mayfield. 